\title{
An EOQ Inventory Model for Time-varying Deteriorating Items with Cubic Demand under Salvage Value and Shortages
}

\author{
Biswaranjan Mandal \\ Department of Mathematics, Acharya Jagadish Chandra Bose College, Kolkata, West Bengal, India \\ Email address: \\ drbrmajcbc@gmail.com

\section{To cite this article:} \\ Biswaranjan Mandal. An EOQ Inventory Model for Time-varying Deteriorating Items with Cubic Demand Under Salvage Value and \\ Shortages. International Journal of Systems Science and Applied Mathematics. Vol. 5, No. 4, 2020, pp. 36-42. \\ doi: 10.11648/j.jijssam.20200504.11
}

Received: October 3, 2020; Accepted: October 17, 2020; Published: November 11, 2020

\begin{abstract}
In this paper, an Economic Order Quantity (EOQ) inventory model is developed for time-varying deteriorating items. Researchers are constantly developing deteriorating inventory models to become more realistic. Many items like paddy, wheat, potato, onion, radioactive substance etc. are becoming damage over time. So time dependent deterioration is more realistic than a constant rate of deterioration of goods used in the present market. The assumption of constant demand rate may not be always appropriate for many inventory items like milk, vegetables etc, the age of these items has a negative impact on demand dure to loss of quality of such products, on the other hand, demand is becoming increased initially when new branded fashionable products like cosmetics, mobile, computer etc are launched in the market. So the demand rate is considered as a cubic function of time and time dependent holding cost. We also want to give importance on salvage value of an inventory system. The model is solved with salvages value associated to the units deteriorating during the cycle. Shortages are allowed and fully backlogged. Finally the model is illustrated with the help of a numerical example, some particular cases are derived and a comparative study of the optimal solutions towards different nature of demand is also presented graphically.
\end{abstract}

Keywords: Inventory, EOQ, Deteriorating Items, Cubic Demand, Salvages Value and Shortages

\section{Introduction}

The traditional inventory model considers the ideal case in which depletion of inventory is caused by a constant demand rate. However, in real life situations there is inventory loss by deterioration also. Study in this direction began with the work of Whitin [1] who considered fashion goods deteriorating at the end of a prescribed storage period. Ghare and Schrader [2] developed a model assuming a constant rate of deterioration. Later many researchers like Shah and Jaiswal [3], Aggarwal [4] etc developed the model with constant demand rate and deteriorating items. But the commonly used items like fruits, meet, food grains (i.e. paddy, wheat, potato, onion etc), perfumes, alcohol, gasoline, radioactive substances etc are becoming more damage over time. So it is more realistic to consider time-varying deteriorating rates.

The assumption of a constant demand rate may not be always appropriate for many inventory items. For example milk, vegetables etc., the age of inventory has a negative impact on demand due to loss of consumer confidence on the quality of such products. So many researchers like Biswaranjan Mandal [5], Mishra and Singh [6], Yadav et al [7], Venkateswarlu and Mohan [8] etc are names to only fewto develop many inventory models..

Again Poonam Mishra and Shah [9] studied an EOQ model for inventory management of time dependent deteriorating items with salvage value. Ajanta Roy [10] developed an inventory model for deteriorating items with price dependent demand and time-varying holding cost. Jaggi et al [11] studied an EOQ model for deteriorating items with salvage value assuming deterioration and demand rate in constant behavior. Karthikeyan et al [12] developed a model to determine the optimum order quantity for constant deteriorating items with cubic demand and salvage value. Their model does not allow for time-varying deterioration and shortages, which would not make applicable in real 
word. Many researchers like Sharma et al [13], Santhi et al. [14], Pakhira et al. [15] etc are noteworthy.

For these sort of situations, efforts have been made to develop a realistic inventory model with time-varying deterioration rate. The demand rate is considered as a cubic function of time and time dependent holding cost. The model is solved with salvages value associated to the units deteriorating during the cycle. Shortages are allowed and fully backlogged. Finally the model is illustrated with the help of a numerical example, some particular cases are derived and a comparative study of the optimal solutions towards different nature of demand is also presented graphically.

\section{Notations and Assumptions}

The mathematical models are developed under the following notations and assumptions:

Notations:

(i) $\mathrm{R}(\mathrm{t})$ : Demand rate.

(ii) $\theta(t)$ : Time-varying deterioration rate.

(iii) $\mathrm{T}$ : The fixed length of each production cycle.

(iv) $\mathrm{HC}$ : Holding cost per unit time

(v) $C_{1}$ : The unit cost of an item.

(vi) DC: Deterioration cost per unit per unit time.

(vii) OC: Ordering cost per order.

(viii)SV: Salvage value per unit per unit time

(ix) $C_{2}:$ Shortage cost unit per unit time.

(x) $\mathrm{I}(\mathrm{t})$ : The inventory level at time $\mathrm{t}$.

(xi) Q: The maximum inventory level during the cycle.

(xii) $\mathrm{T}$ : The length of cycle time.

(xiii)TC: Average total cost per unit time.

Assumptions:

(i). The inventory system included only one item.

(ii). The demand rate is time dependent cubic function

$$
\mathrm{R}(\mathrm{t})=\mathrm{a}+\mathrm{bt}+\mathrm{c} t^{2}+\mathrm{d} t^{3}, \mathrm{a}, \mathrm{b}, \mathrm{c}, \mathrm{d} \geq 0
$$

where $a$ is the initial demand rate, $b$ is the initial rate of change of demand, $\mathrm{c}$ is the rate at which the demand rate increases and $\mathrm{d}$ is the rate at which the change in the demand rate itself increases.

(iii). The time-varying deterioration rate is given by

$$
\begin{gathered}
\theta(t)=\theta_{o} t, 0 \leq \theta_{o}<<1 \\
\mathrm{I}(\mathrm{t})=a\left(t_{1}-t\right)+\frac{b}{2}\left(t_{1}^{2}-t^{2}\right)+\frac{c}{3}\left(t_{1}^{3}-t^{3}\right)+\frac{d}{4}\left(t_{1}^{4}-t^{4}\right)+\frac{a \theta_{o}}{6}\left(t_{1}^{3}-3 t_{1} t^{2}+2 t^{3}\right) \\
+\frac{b \theta_{o}}{8}\left(t_{1}^{4}-2 t^{2} t^{2}+t^{4}\right)+\frac{c \theta_{o}}{30}\left(3 t_{1}^{5}-5 t^{3} t^{2}+2 t^{5}\right)+\frac{d \theta_{o}}{24}\left(2 t_{1}^{6}-3 t^{4} t^{2}+t^{6}\right), 0 \leq t \leq t_{1} \\
\text { And } \mathrm{I}(\mathrm{t})=a\left(t_{1}-t\right)+\frac{b}{2}\left(t_{1}^{2}-t^{2}\right)+\frac{c}{3}\left(t_{1}^{3}-t^{3}\right)+\frac{d}{4}\left(t_{1}^{4}-t^{4}\right), t_{1} \leq t \leq T
\end{gathered}
$$

Since $\mathrm{I}(0)=\mathrm{Q}$, we get from equation (6) the following expression

$$
\mathrm{Q}=a t_{1}+\frac{b}{2} t_{1}^{2}+\left(\frac{c}{3}+\frac{a \theta_{o}}{6}\right) t_{1}^{3}+\left(\frac{d}{4}+\frac{b \theta_{o}}{8}\right) t_{1}^{4}+\frac{c \theta_{o}}{10} t_{1}^{5}+\frac{d \theta_{o}}{12} t_{1}^{6}
$$


Now the average total cost per unit time consists of the following:

Ordering Cost (OC) During the Cycle $[0, \mathrm{~T}]=\boldsymbol{A}$ (Fixed)

Holding cost $(\mathrm{HC})=\int_{0}^{t_{1}}(\alpha+\beta \boldsymbol{t}) \boldsymbol{I}(\boldsymbol{t}) d t$

$$
\begin{aligned}
& =\int_{0}^{t_{1}} \alpha I(t) d t+\int_{0}^{t_{1}} \beta t I(t)=\left[\frac{a \alpha}{2} t_{1}^{2}+\frac{b \alpha}{3} t_{1}^{3}+\frac{c \alpha}{4} t_{1}^{4}+\frac{8 \alpha}{5} t_{1}^{5}+\frac{\theta_{0} a \alpha}{12} t_{1}^{4}+\frac{\theta_{0} b \alpha}{15} t_{1}^{5}+\frac{\theta_{0} c \alpha}{18} t_{1}^{6}+\frac{\theta_{0} d \alpha}{21} t_{1}^{7}\right] \\
& +\left[\frac{a \beta}{6} t_{1}^{3}+\frac{b \beta}{8} t_{1}^{4}+\frac{\beta}{10}\left(c+\frac{\theta_{0} a}{4}\right) t_{1}^{5}+\frac{\beta}{12}\left(d+\frac{\theta_{0} b}{4}\right) t_{1}^{6}+\frac{\theta_{0} c \beta}{56} t_{1}^{7}+\frac{\theta_{0} d \beta}{64} t_{1}^{8}\right] \\
& =\frac{a \alpha}{2} t_{1}^{2}+\left(\frac{b \alpha}{3}+\frac{a \beta}{6}\right) t_{1}^{3}+\left(\frac{c \alpha}{4}+\frac{b \beta}{8}+\frac{a \alpha \theta_{o}}{12}\right) t_{1}^{4}+\left(\frac{d \alpha}{5}+\frac{c \beta}{10}+\frac{b \alpha \theta_{o}}{15}+\frac{a \beta \theta_{o}}{40}\right) t_{1}^{5} \\
& +\left(\frac{d \beta}{12}+\frac{c \alpha \theta_{o}}{18}+\frac{b \beta \theta_{o}}{48}\right) t_{1}^{6}+\left(\frac{d \alpha \theta_{o}}{21}+\frac{c \beta \theta_{o}}{56}\right) t_{1}^{7}+\frac{d \beta \theta_{o}}{64} t_{1}^{8}
\end{aligned}
$$

Cost due to Deterioration $(C D)=C_{1}\left\{I(0)-\int_{0}^{t_{1}} R(t) d t\right\}==C_{1}\left\{\frac{a \theta_{o}}{6} t_{1}^{3}+\frac{b \theta_{o}}{8} t_{1}^{4}+\frac{c \theta_{o}}{10} t_{1}^{5}+\frac{d \theta_{o}}{12} t_{1}^{6}\right\}$

Since Salvage Cost (SV) Is Associated to the Units of Deteriorating During the Cycle [0,T], We get

$$
\text { Salvage cost }(\mathrm{SV})=\mathrm{k} C_{1}\left\{\frac{a \theta_{o}}{6} t_{1}^{3}+\frac{b \theta_{o}}{8} t_{1}^{4}+\frac{c \theta_{o}}{10} t_{1}^{5}+\frac{d \theta_{o}}{12} t_{1}^{6}\right\}
$$

Cost due to Shortage $(C S)=C_{2} \int_{t_{1}}^{T}(T-t) R(t) d t=\int_{t_{1}}^{T}(T-t)\left(a+b t+c t^{2}+d t^{3}\right) d t$

$$
\begin{gathered}
=\int_{t_{1}}^{T}\left\{a(T-t)+b\left(T t-t^{2}\right)+c\left(T t^{2}-t^{3}\right)+d\left(T t^{3}-t^{4}\right)\right\} d t \\
=C_{2}\left\{\frac{a}{2}\left(T^{2}-2 T t_{1}+t_{1}^{2}\right)+\frac{b}{6}\left(T^{3}-3 T t_{1}^{2}+2 t_{1}^{3}\right)+\frac{c}{12}\left(T^{4}-4 T t_{1}^{3}+3 t_{1}^{4}\right)+\frac{d}{20}\left(T^{5}-5 T t_{1}{ }^{4}+4 t_{1}{ }^{5}\right)\right\}
\end{gathered}
$$

The average total cost per unit time of the system during the cycle $[0, \mathrm{~T}]$ will be

$$
\begin{gathered}
\mathrm{TC}\left(t_{1}\right)=\frac{1}{T}[\mathrm{OC}+\mathrm{HC}+\mathrm{CD}-\mathrm{SV}+\mathrm{CS}] \\
=\frac{1}{T}\left[A+\frac{a \alpha}{2} t_{1}^{2}+\left(\frac{b \alpha}{3}+\frac{a \beta}{6}\right) t_{1}^{3}+\left(\frac{c \alpha}{4}+\frac{b \beta}{8}+\frac{a \alpha \theta_{o}}{12}\right) t_{1}^{4}+\left(\frac{d \alpha}{5}+\frac{c \beta}{10}+\frac{b \alpha \theta_{o}}{15}+\frac{a \beta \theta_{o}}{40}\right) t_{1}^{5}\right. \\
+\left(\frac{d \beta}{12}+\frac{c \alpha \theta_{o}}{18}+\frac{b \beta \theta_{o}}{48}\right) t_{1}^{6}+\left(\frac{d \alpha \theta_{o}}{21}+\frac{c \beta \theta_{o}}{56}\right) t_{1}^{7}+\frac{d \beta \theta_{o}}{64} t_{1}^{8}+C_{1}\left\{\frac{a \theta_{o}}{6} t_{1}^{3}+\frac{b \theta_{o}}{8} t_{1}^{4}+\frac{c \theta_{o}}{10} t_{1}^{5}+\frac{d \theta_{o}}{12} t_{1}^{6}\right\} \\
k C_{1}\left\{\frac{a \theta_{o}}{6} t_{1}^{3}+\frac{b \theta_{o}}{8} t_{1}^{4}+\frac{c \theta_{o}}{10} t_{1}^{5}+\frac{d \theta_{o}}{12} t_{1}^{6}\right\}+C_{2}\left\{\frac{a}{2}\left(T^{2}-2 T t_{1}+t_{1}{ }^{2}\right)+\frac{b}{6}\left(T^{3}-3 T t_{1}{ }^{2}+2 t_{1}^{3}\right)\right. \\
\left.\left.+\frac{c}{12}\left(T^{4}-4 T t_{1}^{3}+3 t_{1}^{4}\right)+\frac{d}{20}\left(T^{5}-5 T t_{1}{ }^{4}+4 t_{1}{ }^{5}\right)\right\}\right]
\end{gathered}
$$

For minimum, the necessary condition is $\frac{d T C\left(t_{1}\right)}{d t_{1}}=0$ 
This gives $a C_{1}+\left(b C_{1}+a \alpha\right) t_{1}+\left\{c C_{1}+b \alpha+\frac{a \beta}{2}+\frac{(2-k) a C_{1} \theta_{o}}{2}\right\} t_{1}^{2}+$

$$
\begin{aligned}
\left\{d C_{1}+\right. & \left.c \alpha+\frac{b \beta}{2}+\frac{a \alpha \theta_{o}}{3}+\frac{(2-k) b C_{1} \theta_{o}}{2}\right\} t_{1}^{3}+\left\{d \alpha+\frac{c \beta}{2}+\frac{b \alpha \theta_{o}}{3}+\frac{a \beta \theta_{o}}{8}+\frac{(2-k) c C_{1} \theta_{o}}{2}\right\} t_{1}^{4} \\
+ & \left\{\frac{d \beta}{2}+\frac{c \alpha \theta_{o}}{3}+\frac{b \beta \theta_{o}}{8}+\frac{(2-k) d C_{1} \theta_{o}}{2}\right\} t_{1}^{5}+\left\{\frac{d \alpha \theta_{o}}{3}+\frac{c \beta \theta_{o}}{8}\right\} t_{1}^{6}+\frac{d \beta \theta_{o}}{8} t_{1}^{7} \\
+ & C_{2}\left\{a\left(t_{1}-T\right)+b t_{1}\left(t_{1}-T\right)+c t_{1}^{2}\left(t_{1}-T\right)+d t_{1}^{3}\left(t_{1}-T\right)\right\}=0
\end{aligned}
$$

For minimum the sufficient condition $\frac{\mathrm{d}^{2} \mathrm{TC}\left(\mathrm{t}_{1}\right)}{\mathrm{dt}_{1}{ }^{2}}>0$ would be satisfied.

Let $t_{1}=t_{1}^{*}$ be the optimum value of $t_{1}$.

The optimal values $Q^{*}$ of $\mathrm{Q}$ and $T C^{*}$ of TC are obtained by putting the value $t_{1}=t_{1}^{*}$ from the expressions (8) and (9).

\section{Particular Cases}

(a). If the demand rate is quadratic function of time then $d=0$

From (8), the total amount of inventory $\mathrm{Q}$ becomes

$$
\mathrm{Q}=a t_{1}+\frac{b}{2} t_{1}^{2}+\left(\frac{c}{3}+\frac{a \theta_{o}}{6}\right) t_{1}^{3}+\frac{b \theta_{o}}{8} t_{1}^{4}+\frac{c \theta_{o}}{10} t_{1}^{5}
$$

From (9), the average total cost per unit time of the system during the cycle $[0, T]$ becomes

$$
\begin{gathered}
\operatorname{TC}\left(t_{1}\right)=\frac{1}{T}\left[\mathrm{~A}+\frac{a \alpha}{2} t_{1}^{2}+\left(\frac{b \alpha}{3}+\frac{a \beta}{6}\right) t_{1}^{3}+\left(\frac{c \alpha}{4}+\frac{b \beta}{8}+\frac{a \alpha \theta_{o}}{12}\right) t_{1}^{4}+\left(\frac{c \beta}{10}+\frac{b \alpha \theta_{o}}{15}+\frac{a \beta \theta_{o}}{40}\right) t_{1}^{5}\right. \\
+\left(\frac{c \alpha \theta_{o}}{18}+\frac{b \beta \theta_{o}}{48}\right) t_{1}^{6}+\frac{c \beta \theta_{o}}{56} t_{1}^{7}+C_{1}\left\{\frac{a \theta_{o}}{6} t_{1}^{3}+\frac{b \theta_{o}}{8} t_{1}^{4}+\frac{c \theta_{o}}{10} t_{1}^{5}\right\}-k C_{1}\left\{\frac{a \theta_{o}}{6} t_{1}^{3}+\frac{b \theta_{o}}{8} t_{1}^{4}+\frac{c \theta_{o}}{10} t_{1}^{5}\right\} \\
\left.+C_{2}\left\{\frac{a}{2}\left(T^{2}-2 T t_{1}+t_{1}^{2}\right)+\frac{b}{6}\left(T^{3}-3 T t_{1}^{2}+2 t_{1}^{3}\right)+\frac{c}{12}\left(T^{4}-4 T t_{1}^{3}+3 t_{1}^{4}\right)\right\}\right]
\end{gathered}
$$

The equation (10) becomes

$$
\begin{gathered}
a C_{1}+\left(b C_{1}+a \alpha\right) t_{1}+\left\{c C_{1}+b \alpha+\frac{a \beta}{2}+\frac{(2-k) a C_{1} \theta_{o}}{2}\right\} t_{1}^{2}+ \\
\left\{c \alpha+\frac{b \beta}{2}+\frac{a \alpha \theta_{o}}{3}+\frac{(2-k) b C_{1} \theta_{o}}{2}\right\} t_{1}^{3}+\left\{\frac{c \beta}{2}+\frac{b \alpha \theta_{o}}{3}+\frac{a \beta \theta_{o}}{8}+\frac{(2-k) c C_{1} \theta_{o}}{2}\right\} t_{1}^{4} \\
+\left\{\frac{c \alpha \theta_{o}}{3}+\frac{b \beta \theta_{o}}{8}\right\} t_{1}^{5}+\frac{c \beta \theta_{o}}{8} t_{1}^{6}+C_{2}\left\{a\left(t_{1}-T\right)+b t_{1}\left(t_{1}-T\right)+c t_{1}^{2}\left(t_{1}-T\right)\right\}=0
\end{gathered}
$$

This gives the optimum value of $t_{1}$.

(b). If the demand rate is linear trended function of time then $\mathrm{c}=0$ and $d=0$

From (8), the total amount of inventory $\mathrm{Q}$ becomes

$$
\mathrm{Q}=a t_{1}+\frac{b}{2} t_{1}^{2}+\frac{a \theta_{o}}{6} t_{1}^{3}+\frac{b \theta_{o}}{8} t_{1}^{4}
$$

From (9), the average total cost per unit time of the system during the cycle $[0, T]$ becomes

$$
\mathrm{TC}\left(t_{1}\right)=\frac{1}{T}\left[\mathrm{~A}+\frac{a \alpha}{2} t_{1}^{2}+\left(\frac{b \alpha}{3}+\frac{a \beta}{6}\right) t_{1}^{3}+\left(\frac{b \beta}{8}+\frac{a \alpha \theta_{o}}{12}\right) t_{1}^{4}+\left(\frac{b \alpha \theta_{o}}{15}+\frac{a \beta \theta_{o}}{40}\right) t_{1}^{5}+\frac{b \beta \theta_{o}}{48} t_{1}^{6}\right.
$$




$$
\left.+C_{1}\left\{\frac{a \theta_{o}}{6} t_{1}^{3}+\frac{b \theta_{o}}{8} t_{1}^{4}\right\}-k C_{1}\left\{\frac{a \theta_{o}}{6} t_{1}^{3}+\frac{b \theta_{o}}{8} t_{1}^{4}\right\}+C_{2}\left\{\frac{a}{2}\left(T^{2}-2 T t_{1}+t_{1}^{2}\right)+\frac{b}{6}\left(T^{3}-3 T t_{1}^{2}+2 t_{1}^{3}\right)\right\}\right]
$$

The equation (10) becomes

$$
\begin{gathered}
a C_{1}+\left(b C_{1}+a \alpha\right) t_{1}+\left\{b \alpha+\frac{a \beta}{2}+\frac{(2-k) a C_{1} \theta_{o}}{2}\right\} t_{1}^{2} \\
+\left\{\frac{b \beta}{2}+\frac{a \alpha \theta_{o}}{3}+\frac{(2-k) b C_{1} \theta_{o}}{2}\right\} t_{1}^{3}+\left\{\frac{b \alpha \theta_{o}}{3}+\frac{a \beta \theta_{o}}{8}\right\} t_{1}^{4}+\frac{b \beta \theta_{o}}{8} t_{1}^{5}+C_{2}\left\{a\left(t_{1}-T\right)+b t_{1}\left(t_{1}-T\right)\right\}=0
\end{gathered}
$$

This gives the optimum value of $t_{1}$.

(c). If the demand rate is constant then $\mathrm{b}=0, \mathrm{c}=0$ and $d=0$

From (8), the total amount of inventory $\mathrm{Q}$ becomes

$$
\mathrm{Q}=a t_{1}+\frac{a \theta_{o}}{6} t_{1}^{3}
$$

From (9), the average total cost per unit time of the system during the cycle $[0, \mathrm{~T}]$ becomes

$$
\left.\mathrm{TC}\left(t_{1}\right)=\frac{1}{T}\left[\mathrm{~A}+\frac{a \alpha}{2} t_{1}^{2}+\frac{a \beta}{6} t_{1}^{3}+\frac{a \alpha \theta_{o}}{12} t_{1}^{4}+\frac{a \beta \theta_{o}}{40} t_{1}^{5}+C_{1} \frac{a \theta_{o}}{6} t_{1}^{3}-k C_{1} \frac{a \theta_{o}}{6} t_{1}^{3}+C_{2} \frac{a}{2}\left(T^{2}-2 T t_{1}+t_{1}^{2}\right)\right\}\right]
$$

The equation (10) becomes

$$
a C_{1}+a \alpha t_{1}+\left\{\frac{a \beta}{2}+\frac{(2-k) a C_{1} \theta_{o}}{2}\right\} t_{1}^{2}+\frac{a \alpha \theta_{o}}{3} t_{1}^{3}+\frac{a \beta \theta_{o}}{8} t_{1}^{4}+C_{2} a\left(t_{1}-T\right)=0
$$

This gives the optimum value of $t_{1}$.

\section{Numerical Example}

To illustrate the developed inventory model, let the values of parameters be as follows:

$\mathrm{A}=\$ 500$ per order; $\mathrm{a}=25 ; \mathrm{b}=20 ; \mathrm{c}=10 ; \mathrm{d}=3 ; \theta_{o}=$ $0.02 ; \alpha=0.5 ; \beta=0.6 ; \mathrm{k}=0.1 ; C_{1}=\$ 5$ per unit; $C_{2}=\$ 10$ per unit; $\mathrm{T}=1$ year

Solving the equation (10) with the help of computer using the above parameter values, we find the following optimum outputs $t_{1}^{*}=0.47$ year; $Q^{*}=14.28$ units and $T C^{*}=$ Rs 636.72

It is checked that this solution satisfies the sufficient condition for optimality.

\section{Comparison of Inventory Models Between Varying Demand Rates}

The comparative study is also furnished to illustrate the particular cases of the inventory model by varying demand rates.

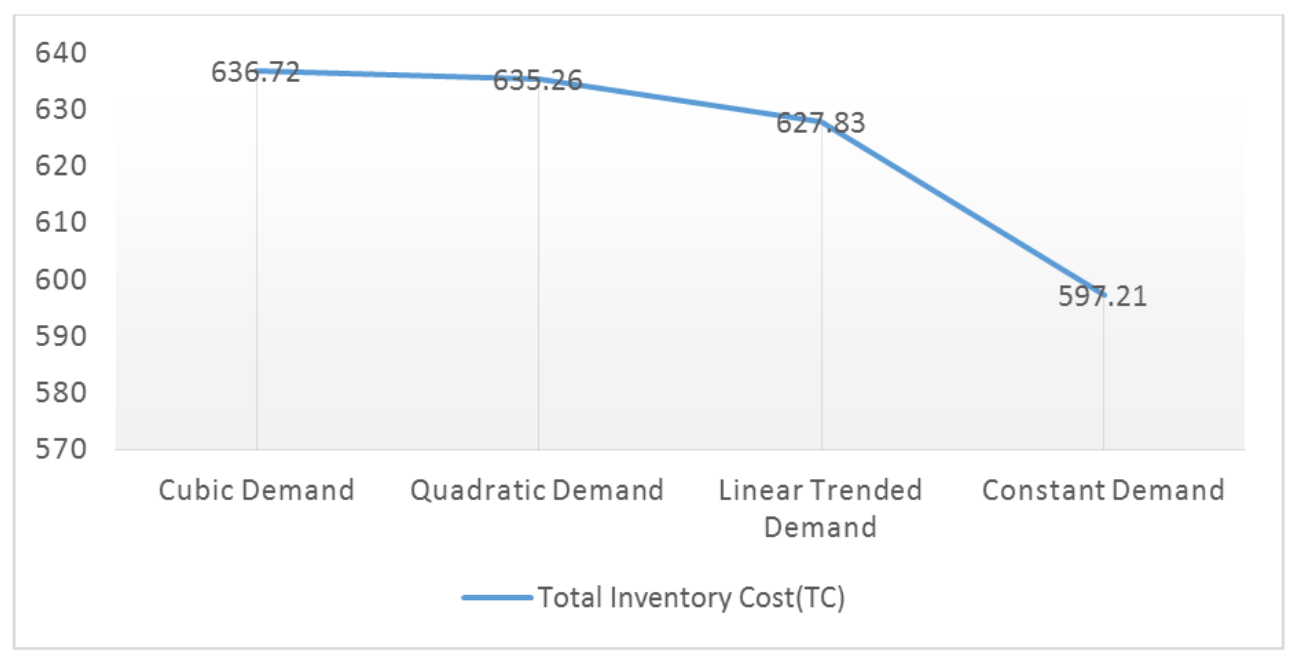

Figure 1. Demand Rate vs OptimalInventory Total Cost. 


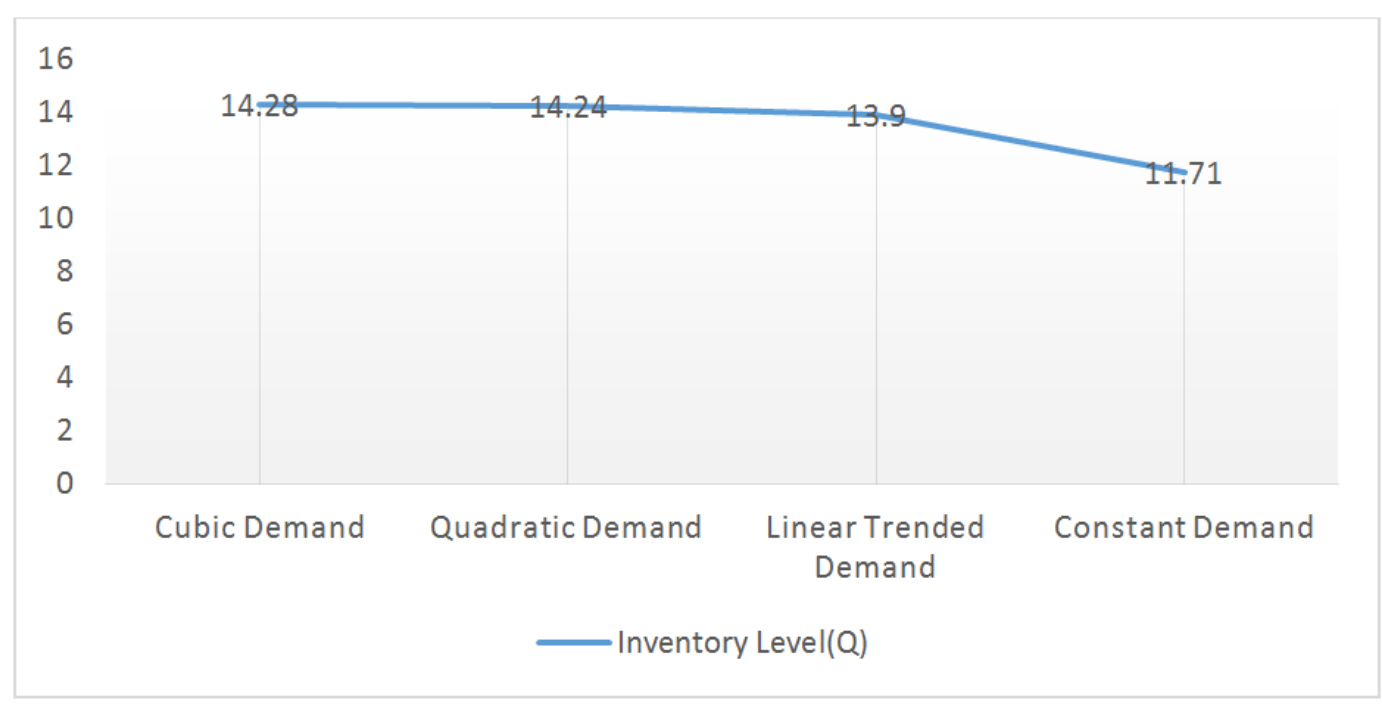

Figure 2. Demand Rate vs Optimal Inventory Level.

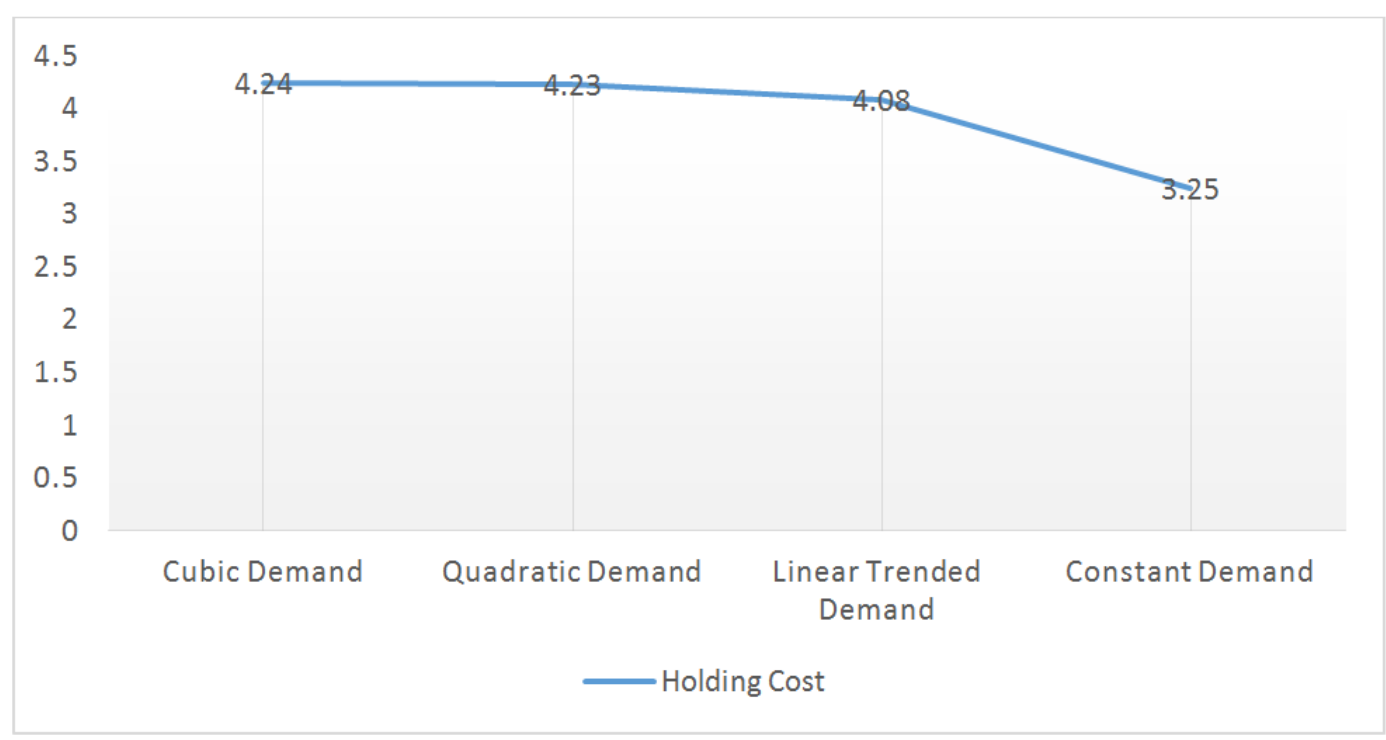

Figure 3. Demand Rate vs Holding Cost.

\section{Concluding Remarks}

In this study, an EOQ model has been framed for timevarying deteriorating items under cubic demand in nature. The salvage value has been incorporated for deteriorating items with time dependent holding cost. Shortages are allowed which are fully backlogged. The models are developed analytically as well as computationally with graphical representation.

Efforts are given on comparative study graphically between optimal inventory total cost, optimal inventory level and holding cost considering cubic, quadratic, linear and constant nature of demand rates. Analyzing Figure 1, Figure 2 and Figure 3, it is observed that optimality of inventory total cost, inventory level and holding cost are moderately changing for cubic, quadratic and linear trended demand rates, where as these are changing significantly towards the model with constant demand in nature.

\section{References}

[1] T. M. Whitin, “Theory of inventory management", Princeton University Press, USA., (1957).

[2] P. M. Ghare and G. F. Schrader, "A model for exponential decayinginventory. Journal of Industrial Engineering, 14, (1963), pp. 238-243.

[3] Y. K. Shah and M. C. Jaiswal, "Anperiodic review inventory model for items that deteriorate continuously in time", Int. J Prod. Res., 15, 1977, pp. 179-190.

[4] S. P. Aggarwal, "A note on an order-level inventory model for a system with constant rate of deterioration", Opsearch, 15, (1978), pp. 184-187.

[5] Biswaranjan Mandal, "An EOQ inventory model for Weibull distributed deteriorating items under ramptype demand and shortages", Opsearch, 47 (2), 2010, pp. 158-166. 
[6] V. Mishra and L. Singh, "An inventory model for ramptype demand, time dependent deteriorating items with salvage value and shortages, Int. J Appl. and Maths and Statistics, 23 (11), 2011, pp. 84-91.

[7] R. K. Yadav and P. Yadav, "Volume flexibility in production model with cubic demand rate and Weibull distribution with partial backlogging", ISOR J. of Mathematics, 4, 2013, pp. 29-34.

[8] R. Venkateswarlu and R. Mohan, "An inventory model with quadratic demand, constant deterioration and salvage value, Res. J. of Mathematical and Statistical Sciences", 2 (1), 2014, pp. 1-5.

[9] Poonam Mishra and N. H. Shah, "Inventory management of time dependent deteriorating items with salvage value", Applied Mathematical Sciences, 16 (2), 2008, pp. 793-796.

[10] Ajanta Roy, "An inventory model for deteriorating items with price dependent demand and time-varying holding cost", AMO, 10 (1). 2008.

[11] C. K. Jaggi and S. P., "Aggarwal. EOQ for deteriorating items with salvage value", Bulletin of Pure and Applied Sciences, 15 (1), 1996, pp. 67-71.

[12] K. Karthikeyan and G. Santhi, "An inventory model for constant deteriorating items with cubic demand and salvage value", Int. J Appl Eng. And Res., 10 (55), 2015 pp. 37233728 .

[13] V. Sharma, A. K. Sharma, " A Deterministic Inventory Model with Cubic Demand and Infinite Time Horizon with Constant Deterioration and Salvage Value", International Journal of Science and Research (IJSR), 5 (11), 2016, pp. 1643-1646.

[14] G Santhi and K Karthikeyan, "An EOQ model for Weibull distribution deterioration with time-dependent cubic demand and backlogging", IOP Conference Series: Materials Science and Engineering, 263 (2017) 042132 doi: 10.1088/1757$899 \mathrm{X} / 263 / 4 / 042132$.

[15] R. Pakhira, UttamGhosh and S. Sarkar, "Study of Memory Effect in an Inventory Model with Quadratic Type Demand Rate and Salvage Value", Applied Mathematical Sciences, 13 (5), 2019, pp. 209-223. 